\title{
EL PROCESO DE ATENCIÓN DE ENFERMERÍA
}

\section{Conocimientos al respecto en la Facultad de Enfermería de la Fundación Universitaria de Ciencias de la Salud}

\author{
María Isabel Camacho, Jeimy Chávez, Jacqueline Otero, Adriana Carolina Rodríguez*
}

\section{Resumen}

El objeto de esta investigación es determinar si existen dificultades en la aplicación del Proceso de Atención de Enfermería (PAE) por parte de los estudiantes de segundo a octavo semestre de la Facultad de Enfermería de la Fundación Universitaria de Ciencias de la Salud, en el período comprendido entre agosto y septiembre de 2000. Para ello se llevó a cabo un estudio cuali-cuantitativo, descriptivo, transversal, mediante la aplicación de un cuestionario estructurado. Los resultados mostraron que los estudiantes de enfermería presentan dificultades para aplicar el PAE pues a pesar de tener el conocimiento teórico, no es lo suficientemente sólido como para llevarlo a la práctica; además, el tiempo dedicado para el estudio de dicho proceso es insuficiente, tanto por parte del alumno como de la Facultad de Enfermería, hay incongruencia entre el nivel de conocimientos de los docentes y los estudiantes están desmotivados para aplicar el PAE.

\section{Generalidades}

El propósito de esta investigación es determinar si existen dificultades en la aplicación del Proceso de Atención de Enfermería (PAE), por parte de los estudiantes de segundo a octavo semestre de la Facultad de Enfermería de la Fundación Universitaria de Ciencias de la Salud (FUCS) para que los resultados del análisis de datos sean conocidos por las directivas de la Facultad de Enfermería y así contribuir para tomar las medidas correctivas y se sigan promoviendo profesionales de servicio que se fundamenten en el "Proceso de Enfermería" para brindar cuidado integral a la persona, familia y comunidad.

La historia muestra el proceso evolutivo de la enfermería paralelo a la consolidación del proceso de "Atención de Enfermería" como herramienta fundamental del cuidado brindado por el profesional de enfermería; por esta razón la facultad de Enfer-

Séptimo semestre, Facultad de Enfermería de la Fundación Universitaria de Ciencias de la Salud, FUCS. Bogotá, D.C. mería de la Fundación Universitaria de Ciencias de la Salud, tiene como objetivo que el egresado de enfermería demuestre en la práctica capacidades y conocimientos para aplicar el PAE y proporcione cuidado directo, integral y continuado a la madre, el niño, el adulto y el anciano en diferentes situaciones de salud.

Un recorrido por la evolución del PAE ubica su origen en 1955 con Hall, y desarrollos posteriores en 1959 por Johnson, Orlando en 1961 y Wiedenbach en 1963, quienes consideraban sólo tres fases en el proceso: valoración, planificación y ejecución.

Para 1967 Yura y Walsh, implementaron la fase de evaluación, y finalmente, la de diagnóstico fue añadida por Bolch en 1974 y Roy en 1975, consolidando así un proceso con cinco fases. Desde este momento el PAE fue definido como un método sistemático, organizado, que permite brindar un cuidado eficaz y eficiente orientado al logro de objeti- 
vos, y fue legitimado como un sistema de la práctica de Enfermería por la Asociación de Enfermeras Americanas, ANA.

Con ayuda de la investigación realizada por Mary Giraldo de Bustos en 1975 se concluyó acerca del que PAE: "Se debe iniciar la enseñanza desde el comienzo de la carrera, implantando una cátedra donde se haga énfasis en el planteamiento del cuidado por medio de la unificación de la metodología. De la misma manera dedicar más tiempo, conservando lo básico para permitir que sea más práctico y con menos complicación".

Otro estudio acerca del PAE realizado por Reyes Reyes, Quintero y Crispín muestra que entre los factores asociados a la aplicación del PAE están el tipo de servicio, el exceso de trabajo, el tipo de pacientes, profundidad en ciertos procedimientos, interés del personal, interrelación en el personal administrativo y operativo del contexto institucional. Además el estudio reflejó que las enfermeras necesitan actualización y capacitación sobre el manejo de la taxonomía de la NANDA; y se pudo determinar que no solo los estudiantes de enfermería presentan dificultad en la aplicación del PAE sino que también el profesional de enfermería durante su ejercicio práctico todavía presenta dificultades.

\section{Materiales y métodos}

Se utilizó una clase de muestreo aleatorio estratificado. Se dividió la muestra (195 estudiantes) en subconjuntos homogéneos (por semestres) lo cual permitió alcanzar un mínimo de error.

La recolección de los datos se realizó por medio de un instrumento estructurado, el cual consta de 23 preguntas así: 2 de falso y verdadero; 5 de selección múltiple; 1 de ordenamiento; 5 de preguntas dicotómicas abiertas; 9 apreciaciones acerca de la motivación contenidas en una escala.

El 14 de agosto de 2000 se llevó a cabo una prueba piloto con los estudiantes de séptimo y octavo semestre de la Facultad de Enfermería de la Pontificia Universidad Javeriana, donde fueron entregados 20 instrumentos de recolección de datos, los cuales tu- vieron previamente un proceso de validación realizado pór cinco expertos cuyas correcciones fueron aplicadas en la prueba, y sólo 12 de estos fueron devueltos, con los cuales se realizó el análisis respectivo a cada pregunta y se verificó el nivel de facilidad, dificultad y solidez de las mismas, para hacer los respectivas cambios al instrumento. El día 6 de octubre de 2000, se realizó la recolección de los datos de 195 estudiantes de segundo a octavo semestre de la Facultad de Enfermería de la Fundación Universitaria de Ciencias de la Salud (FUCS)..

El análisis se hizo de forma manual, teniendo en cuenta la categorización de la variable: conocimientos acerca del PAE, tiempo dedicado al estudio de dicho Proceso, unificación de conocimientos por parte de los docentes de la Facultad de Enfermería y motivación intrínseca y extrínseca. Fueron empleados cuadros para presentar el resumen o clasificación de los resultados de las variables del estudio mostrando frecuencias y porcentajes, respectivamente.

\section{Resultados}

Al finalizar el estudio, y después de hacer el análisis completo, se encontró que los estudiantes de segundo a octavo semestre de la Facultad de Enfermería de la FUCS presentan dificultades en la aplicación del PAE.

Así mismo, los estudiantes que presentan mejores conocimientos teóricos acerca del PAE, son los de II y III semestre de enfermería, con un 73,4 y $74,5 \%$ respectivamente. Por otro lado los estudiantes con mayor déficit de conocimientos son los de VI y VIII semestre con $63,3 \%$ y $64,4 \%$ respectivamente (Tabla1).

Los estudiantes de II a VIII semestre dicen que no es suficiente el tiempo dedicado al estudio del Proceso en la teoría, lo cual se refleja en $71,1 \%$ de la muestra (Tabla 2).

Se observa que en II semestre, el 70\% de los estudiantes le dedican tiempo al estudio del PAE, y es lamentable constatar que al llegar a VIII semestre $60 \%$ no lo hacen, sin olvidar que es una responsabilidad del estudiante actualizarse y despejar dudas. Según $81,4 \%$ de la muestra, en el cuerpo docente de la Fa- 


\section{Conocimientos sobre el proceso de atención de enfermería (PAE)}

Opcion de Respuestas

Tiene conocimientos

$\mathbf{F}$

\section{DEFINICION}

\section{Semestre}

Segundo

Tercero

Cuarto

Quinto

Sexto

Séptimo

Octavo

\section{OBJETIVO}

Semestre

Segundo

Tercero

Cuarto

Quinto

Sexto

Septmo

Octavo

\section{ETAPAS DE P.A.E}

\section{Semestre}

Segundo

Tercero

Cuarto

Quinto

Sexto

Séptimo

Octavo
28

26

25

28

22

26

11

23

24

23

25

22

22

10
No tiene conocimientos

$\%$

$76.7 \quad 6$

$80 \quad 6$

$\begin{array}{ll}76.7 & 7\end{array}$

83.35

73.38

73.36

66.75
No responde

TOTAL

\section{$\%$}

93.41

86.74

83.35

93.32

73.38

$86.7 \quad 2$

$73.3 \quad 4$

$50 \quad 15$

$56.7 \quad 13$

$46.7 \quad 16$

$40 \quad 18$

$43.3 \quad 17$

$46.7 \quad 16$

53.37

\subsection{1}

13.3

16.70

6.70

$26.7 \quad 0$

$6.7 \quad 2$

$26.7 \quad 0$
$3.3 \quad 30$

100

100

100

100

100

100

100

\section{Tabla 1}

cultad existe incongruencia en cuanto a los conocimientos acerca del PAE. Es importante anotar que en la Facultad se venían realizando talleres para ellos (Tabla 3).

Por último, los estudiantes de II a VIII semestre están desmotivados para aplicar el PAE, a pesar de que lo consideran importante durante la vida profesional (Tabla 4).

\section{Recomendaciones}

Siendo el PAE la base de la práctica profesional, resulta necesario crear una cátedra del proceso como asignatura para cada semestre.

De igual manera, es necesario establecer objetivos claros respecto al PAE, que permitan aumentar la motivación y el espíritu investigativo del estudiante frente al tema. 


\section{Tiempo dedicado al estudio del proceso de atención de enfermería (PAE)}

\begin{tabular}{|c|c|c|c|c|c|c|c|c|}
\hline \multirow[t]{2}{*}{ Opcion de Respuesta } & \multicolumn{2}{|c|}{ Suficiente } & \multicolumn{2}{|c|}{ No es suficiente } & \multicolumn{2}{|c|}{ No responde } & \multicolumn{2}{|c|}{ TOTAL } \\
\hline & $\mathbf{F}$ & $\%$ & $\mathbf{F}$ & $\%$ & $\mathbf{F}$ & $\%$ & $\mathbf{F}$ & $\%$ \\
\hline \multicolumn{9}{|l|}{ TIEMPO EN TEORÍA } \\
\hline \multicolumn{9}{|l|}{ Semestre } \\
\hline Segundo & 11 & 36.7 & 18 & 60 & 1 & 3.3 & 30 & 100 \\
\hline Tercero & 6 & 20 & 24 & 80 & 0 & 0 & 30 & 100 \\
\hline Cuarto & 9 & 30 & 21 & 70 & 0 & 0 & 30 & 100 \\
\hline Quinto & 3 & 10 & 26 & 86.7 & 1 & 3.3 & 30 & 100 \\
\hline Sexto & 6 & 20 & 23 & 76.7 & 1 & 3.3 & 30 & 100 \\
\hline Séptimo & 6 & 20 & 24 & 80 & 0 & 0 & 30 & 100 \\
\hline Octavo & 2 & 13.3 & 13 & 86.7 & 0 & 0 & 15 & 100 \\
\hline
\end{tabular}

\section{TIEMPO EN PRÁCTICA}

\section{Semestre}

\section{Segundo}

Tercero

Cuarto

Quinto

Sexto

Septmo

Octavo

\section{ÁUTOESTUdiO}

\section{Semestre}

Segundo

Tercero

Cuarto

Quinto

Sexto

Séptimo

Octavo

$\begin{array}{ccc}22 & 73.3 & 8 \\ 12 & 40 & 18 \\ 17 & 56.7 & 13 \\ 19 & 63.3 & 11 \\ 14 & 46.7 & 15 \\ 5 & 16.7 & 25 \\ 5 & 33.3 & 10\end{array}$

10

$\begin{array}{cc}70 & 9 \\ 46.7 & 16 \\ 53.3 & 14 \\ 33.3 & 20 \\ 33.3 & 19 \\ 40 & 17 \\ 40 & 9\end{array}$

$\begin{array}{cc}26.7 & 0 \\ 60 & 0 \\ 43.3 & 0 \\ 36.7 & 0 \\ 50 & 1 \\ 83.3 & 0 \\ 66.7 & 0\end{array}$

o




\section{Unificación de conceptos sobre el (PAE)}

Opcion de Respuesta

\section{TEORÍA Y PRÁCTICA}

\section{Semestre}

Segundo

Tercero

Cuarto

Quinto

Sexto

Séptimo

Octavo

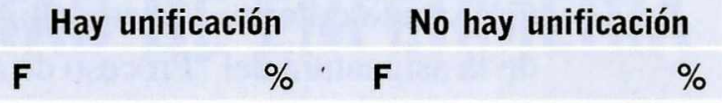

$\%$
No responde

$\mathbf{F}$
TOTAL

\% $\quad$ F $\%$

\section{Tabla 3}

\section{Motivación frente al proceso de atención de enfermería (PAE)}

Apreciaciones

14. Aplicar el PAE motiva al estudiante a mejorar su cuidados de enfermería.

15. Los estímulos recibidos en la teoría por el estudiante de parte del docente acerca del P.A.E. motivan al estudiante a profundizar en el tema.

16. Los estímulos brindados en la práctica por parte del instructor motivan al estudiante a realizar día a día el P.A.E.

17. Los estímulos brindados para estudiar y aplicar el P.A.E. durante la teoría y la práctica debilitan el interés del estudiante.

$13.3 \%$

$20.5 \%$

$35.4 \%$

$40 \%$

$13.9 \%$

$21.5 \%$

$36.4 \%$

$16.9 \%$

$10.2 \%$

$1 \%$
21. El estudiante aplica el P.A.E. por ser una actividad indispensable para alcanzar el título profesional. 


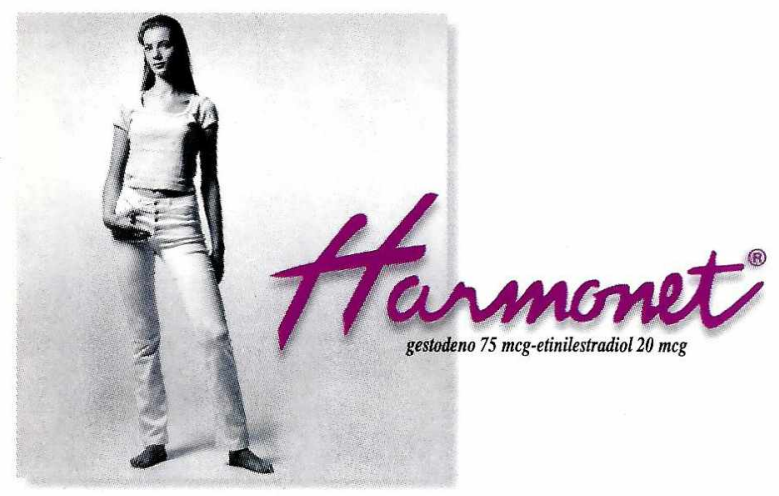

\section{FORMULA}

Cada gragea contiene: Gestodeno $75 \mathrm{mcg}$, Etinilestradiol $20 \mathrm{mcg}$, lactosa $37,155 \mathrm{mg}$, azúcar $19,66 \mathrm{mg}$ almidón de maíz $15,5 \mathrm{mg}$, carbonato de calcio $8,697 \mathrm{mg}$, talco $4,242 \mathrm{mg}$ povidona K-90 $171 \mathrm{mcg}$, cera E $50 \mathrm{mcg}$.

\section{INDICACIONES}

Harmonet está indicado para la prevención del embarazo en mujeres que deciden utilizar este método.

\section{POSOLOGIA Y FORMA DE ADMINISTRACION}

Para lograr una eficacia anticonceptiva óptima, HARMONET deberá ingerirse diariamente según las indicaciones y con intervalos diarios que no excedan las 24 horas. Debe instruirse a la paciente para que tome las grageas todos los dias a la misma hora, preferiblemente con la comida de la noche o bien antes de acostarse.

\section{CONTRAINDICACIONES}

Harmonet está contraindicado en las mujeres que presenten:

Antecedentes o diagnóstico de trastornos trombóticos arteriales o venosos, trastornos embólicos o situaciones que pudieran predisponer a la paciente (por ejemplo, defectos de la coagulación, valvulopatias cardiacas, fibrilación auricular); trastornos agudos o crónicos de la función hepática (ncluyendose entre éstos el sindromé de Dubinjohnson o al sindrome idjoṕtir), antecedentes o diagnóstico de tumores hepáticos antecedentes de ictericia

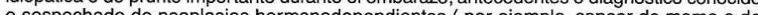
- sospechio): trastornos del momoliodependientes ( por ejemplo, cancer de mama o de

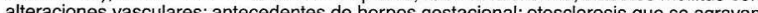
durante el hipersensibilidad a cualquiera de los componentes de Harmonet.

\section{EFECTOS SECUNDARIOS}

Los siguientes efectos secundarios se han registrado en un total de 1338 pacientes que recibieron Harmonet durante el curso de tres estudios clínicos:

Generales Incidencia >3\%: dolor abdominal, dolor de espalda, dolor en los genitales, dolor pélvico. Incidencia < que 1\%: distensión del abdomen, abcesos, reacciones alérgicas, astenia, fiebre, síndrome gripal, pesadez en las extremidades, infecciones, malestar, moniliasis, arttritis reumatoidea. Sistema Cardiovascular. Incidencia $>3 \%$ : migraña. Incidencia de 1\%-3\%: venas varicosas. Incidencia < $1 \%$ : dolor al pecho trombosis de las venas profundas sofocos, hipertension, palpitaciones, taquicardia, tromboembolia, tromboflebitis, vasodilatacion y otros trastornos cardiovasculares. Sistema Digestivo. Incidencia < 3\% Náuseas. Incidencia 1\%-3\%: Vómito. Incidencia < 1\%: colecistis, diarrea, flatulencia, transtornos vesiculares, gastritis, gastroenteritis, transtornos gastrointestinales, hepatopatía, aumento del apetito estomatitis. Sistema Metabólico Incidencia < $1 \%$ edema aumento o pérdida de peso. Sistema Nervioso Incidencia $>3 \%$ Cefaleas, nerviosismo. Incidencia 1\%-3\%: Depresión, mareos, alteraciones en la libido. Incidencia < 1\%: amnesia, ataxia, hostilidad, parestesia, transtornos en el sueño, somnolencia, sudoración excesiva. Sistema Respiratorio. Incidencia $<1 \%$ bronquitis, faringitis, rinitis, sinusitis. Dermatológicos. Incidencia < $1 \%$ : Acne. Incidencia $<1 \%$ : alopecia, cloasma, eczema, prurito, eritema otros transtor dermatol, intus, sordera total transitoria. Sistema Urogenital. Incidencia $>3 \%$. amenorrea, sangrado en los genital, goteo, tensión mamaria. Incidencia <1\%. cistilis, dismenorrea, disuria, dolor del trato urinario sequedad vaginal, moniliasis vaginal vaginitis, transtornos vuluecciones Por otra parte, los siguientes efectos secundarios se han registrado en vulvovaginales. recibian anticonosigus orales y se consideraron relacionados con la droga recibian anticonceptivos orales y se consideraron relacionados con la droga administrada: inflamación), sangrado intermenstrual, goteo, alteraciones en el flujo menstrual, amenorrea, infertilidad temporaria con posterioridad a la descontinuación del tratamiento, edema, melasma que puede persistir, aumento o disminución de peso, alteraciones en la erosión y secreción cervical, disminución en el volumen de leche en el postparto inmediato, iclericia colestática, migraña, eritema (alérgico), depresión mental, menor tolerancia a los carbohidratos, candidiasis vaginal, acentuación de la curvatura de la córnea, intolerancia a los lentes de contacto, cambio en las mamas: tension, aumento de tamano, secreción. Existen evidencias de una posible relación entre los siguientes efectos secunadrios y la utilización de anticonceptivos orales, aunque aún debe confirmarse esta informacion: Trombosis mesentérica, Trombosis retiniana. Los siguientes efectos secunadrios se han registrado en pacientes que recibían anticonceptivos orales, pero su asociación no ha sido aún confirmada ni rechazada: Anomalías congénitas, síndrome premestrual, cataratas, neuritis óptica, cambios en el apetito, síndrome similar a la cistitis, cefaleas, nerviosismo, mareos, hirsutismo, perdida del cabello, eritema multiforme, eritema nodoso, erupción hemorrágica, vaginitis, disminución de la función renal, síndrome urémico hemolítico, síndrome de BuddChiari, acné, cambios en la libido, colitis, enfermedad cerebrovascular, con prolapso de la valvula mitral, sindrome Para maýr informar

SOBREDOSIS

La sobredosis puede causar náuseas o vómitos; en las mujeres puede producirse sangrado por suspresion. anticonceptivos orales.

PRESENTACION

Envase con 21 grageas. Registro INVIMA 006606

\section{Wyeth}

mería de la Fundación Universitaria de Ciencias de la Salud reglamente la capacitación de los docentes respecto al tema, unificando criterios y metodología por medio de la creación de la asignatura del "Proceso de Atención de Enfermería".

\section{Bibliografía}

ANEC. Junta Directiva Nacional. Ley 266 de 1996. El Congreso de Colombia.

Arroyo, A. Cortina. Ética y Legislación en Enfermería. 1998. 29-89.

Cáceres H. Sociedad de Cirugía de Bogotá. 1988; 98-99-101.

Codesa H. Historia de la Enfermería. Editorial Iberoamericana.

De Morrillo G, Garzón N. Departamento de Enfermería Hospital de San José. 1986.

Durán de Villalobos M. Modelo Educativo de Enfermería en Colombia. Revista de Avances en Enfermería 1994; 3: 16-23

Gutiérrez de Reales E. Examen de un Modelo Conceptual de Enfermería. Avances de Enfermería 1985; 5: 75-82

Jamieson ME. Historia de Enfermería.

Lyer P. Proceso y Diagnósticos de Enfermería. Mc. Graw Hill Interamericana. 1997.

Marriner TA. Modelos y Teorías en Enfermería. Editorial Mosby Doima. 1994.

Silva de Mojica C. Remembranza alrededor de 25 años de ACOFAEN. Revista Avances de Enfermería 1995; 13: 19-23.

Wieyr P. Proceso y diagnóstico de Enfermería. Editorial Mc Graw Hill. 1997. 